\title{
A Literature Review of the Human Body as a Communication Medium using RedTacton
}

\author{
Yusuf Perwej, PhD \\ M.Tech, Ph.D (Computer Science \& Engg.) \\ Assistant Professor, Department of Computer Information System \\ Al Baha University, Al Baha, Kingdom of Saudi Arabia (KSA)
}

\begin{abstract}
At present many technologies for networking are known and are in use. These technologies connect people, objects and other networks together so as to dispense data and thus make information ready for access. In the world of computers, networking is the practice of linking two or more computing devices together for the purpose of dispensing data. In this paper, we introduce novel networking technology called RedTacton was announced by NTT labs from Japan in 2005, which makes use of human body surface as communication medium when exchanging data among people, objects and networks. The RedTacton takes a different technical viewpoint. Instead of relying on electromagnetic waves or light waves to carry data, RedTacton uses feeble electric fields on the surface of the body as a transmission medium. Electricfield communication is dissimilar from orthodox wireless or infrared communication which simply does not rely on electromagnetic or light wave to transmit data because it uses the electric field procreate on the surface of the human body. This is mainly developed to conquer an interposing of unwanted signal in intensive places has become a major issue and the better solution to this can be, a human body functioning as non-stop transmitter and receiver. The RedTacton communication is possible using any body surfaces, such as the hands, fingers, arms, feet, face, legs or toes. Red Tacton can be used in any media including water, glass, wood, shoes and clothing as well etc. The electric field/Photonics method employed by Red Tacton is preferable to conventional methods in terms of communication distance, speed, and interactivity. In this paper, I will discuss about RedTacton, and it's working states, body coupled communications, transceiver and applications of RedTacton in various fields.
\end{abstract}

\section{Keywords}

Body Coupled Communications, RedTacton, Intrabody, Electric Field, Capacitive Body Coupling, Transceiver.

\section{INTRODUCTION}

Today, people can communicate anytime, anywhere, and with anyone over a cellular phone network. Additional, the Internet lets people download limitless quantities of data from remotely located servers to their home computers. Indefeasibly, these two technologies enable communications between terminals located at [1] a distance from each other. The all kinds of electronic devices including personal digital assistants (PDAs), pocket video games, and digital cameras are becoming smaller, so people can carry around or even wear various personal information and communication [2] appliances during their day-to-day activities. In spite of, userfriendly ubiquitous services involve more between remotely located terminals. When cables are used for communication between terminals, the routing of cables is clearly inconvenient. When very feeble radio signals are used, data speeds are reduced by unwanted packet collision and other such problems in intensive places such as exhibition sites and security risk from unwanted signal interruption is another problem. All these problems have been overlooked by more substantial and energy efficient technology. Unless, they each have various fundamental technical [3] limitations that constrain their usage, such as the precipitous falloff in transmission speed in multi-user environments producing network congestion [4]

These personal area networks are already possible using radio-based technologies, such as Wi-Fi or Bluetooth, or just plain old cables to connect devices. However, Nippon Telegraph and Telephone (NTT) Corporation, the Japanese communications company [5], has developed a technology called RedTacton, which it claims can send data over the surface of the skin at speeds of up to $2 \mathrm{Mbps}$ identical to a fast broadband data connection. NTT is not the first company to use the human body as a conduit for data. The IBM pioneered the field in 1996 with a system that could transfer small amounts of data at very low speeds, and last June, Microsoft was granted a patent for a method and apparatus for transmitting power and data using the human body. Using the RedTacton, we can establish a communication network with the help of the electrical energy on the surface of the body [6]. The network here increases with the number of users in contact with each other. This forms the cutting edge technology of the upcoming epoch, an epoch ubiquitous computing, when networks are seamlessly interconnected and information is consistently readily available at our fingertips both literally and metaphorically.

Practically RedTacton enabled devices, music from an MP3 player in your pocket would pass through your clothing and shoot over your body to headphones in your ears. Instead of fiddling around with a cable to connect your digital camera to your computer, you could devolution pictures just by touching the PC while the camera is around your neck. And since data can devolution from one body to another [7], you could also commutation electronic business cards by shaking hands, trade music files by dancing cheek to cheek, or swap phone numbers just by kissing. If we could use the human body itself as a transmission medium, then this would be an ideal way of implementing human area networks because it would solve at a thump all the problems including low security, throughput deficiency, and exalted network setup costs. 


\section{RELATED WORK}

The rapid growth of low power integrated circuit, wireless communication such as Bluetooth, ZigBee, Wi-Fi and wireless sensor network has created a new generation of wireless body area network (WBAN). The human body is modeled as an electrical channel for communication by using the concept of capacitive coupling or AC coupling which allows only the AC signal while blocks the DC signal. The body coupled communications was discovered in the middle of the nineties. The MIT (T. G. Zimmerman) [8] discovered body coupled communications by accident while doing human interface research on position sensors. At the same time Sony Labs a similar technology was developed that resulted in the wearable key prototype [9]. These discoveries led to an initial media frenzy. But expeditiously afterwards interest was lost in body coupled communications because of what was then thought were fundamental limitations of the technology. Until the initial stages, there was also research done on powering devices via the body. Yet this did not lead to many . After a period of disinterest in the technology, between 2003- 2006 the technology regained some research interest. At this time different techniques were tried and thus managed to reach higher speeds. The first major breakthrough came from NTT (Nippon Telegraph and Telephone) in 2004. Using an electro optical implementation a speed of $10 \mathrm{Mb} / \mathrm{s}$ was reached. One of their applications was sending video through the body [5]. Nearly the same time the Skinplex technology became available which was a very simple implementation with very low speeds and very low energy consumption. This implementation was not advanced enough for body sensor networks and was mainly used for user identification. A few years later the SSL (Semiconductor System Lab) group from KAIST (Korea Advanced Institute of Science and Technology) was able to present a more power efficient implementation of the body coupled communications using wideband signaling [10]. This implementation has been steadily transcendent with higher data rates and features like interference robustness. The signal transmission and interBCC network interference measurement results have been presented by the authors from Electronics and Telecommunications Research Institute (ETRI) in [11]. The implementation of NTT is slowly moving towards commercialization. The first [12] commercial implementation of their security technology is the NTT Firmo evaluation kit. The implementation from KAIST is still in development in the lab and will probably be commercialized in the near future. Instantaneously it appears that about five [12] research groups are actively pursuing body coupled communications [13]. Two of those groups have working advanced implementations and the others are still experimenting with the basic parameters.

\section{THE REDTACTON}

The RedTacton is a new Human Area Networking technology that uses the surface of the human body as a safe, high speed network transmission path. This technology uses the minute electric field emitted on the surface of the human body. The concept of RedTacton is point to point network [3] transrecievers exchange information none other server receives data using photonic electric field sensors combined with optic crystal \& laser light. The communication is feasible through any part of the human body. In the RedTacton concept, we are transmitting the data stream relates to (Analog or Digital) as an input to the circuitrary, two probes are attached or connected in the contact of human bodies. In this technology, the human body behaves like an optical wire or transmitting media in the system. The communication or data transfer is done because of the [4] electric field which is naturally present in the human body. The human body is the best conductor of the electric field, hence exchange of data is easily acquired. According to fact, it is completely distinct from wireless [14] and infrared. A transmission path is formed at the instant a part of the human body comes in contact with a RedTacton transceiver. The Human Area Networking (HAN) is a technology that safely turns the surface of the human body into a data transmission path at speeds up to $10 \mathrm{Mbps}$ amid any two points on the body. When terminals carried by the user or embedded in devices are linked in various combinations according to the user's communication is possible using any body surfaces, such as the hands, fingers, arms, feet, face, legs or torso[15]. RedTacton works natural, physical movements and it can be used life, temporary one-to-one private networks based

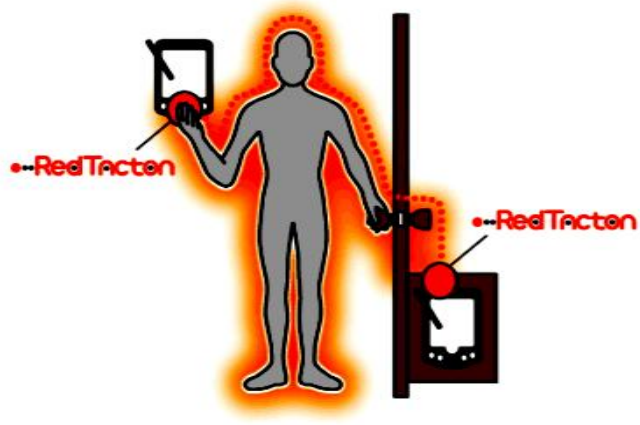

Figure 1. The RedTacton

for intuitive operation of computer-based systems in day to day on individualistic handshaking, device personalization, security, and a host of dissimilar applications based on new behavior patterns enabled by RedTacton.

\section{THE BODY COUPLE COMMUNICATION (BCC) TECHNOLOGY}

In the BCC technology, the human body serves as a communication channel. The basic theory of BCC is that a small electric field is induced into the human body in order to propagate a signal between devices that are in the impedance of [16], or in direct contact with, the human body. The two conceptually different approaches to motivate the electrical signal onto the body have been in advance proposed first capacitive coupling and second galvanic coupling. 


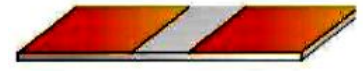

Electrode $\square$ Dielectric

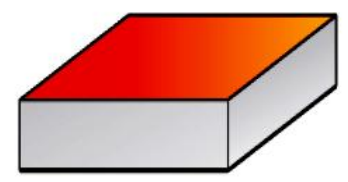

Electrode $\square$ Dielectric (b) Vertical structure.

Figure 2. The Coupler Structure for Capacitive \& Galvanic Body Coupling

These two techniques, the BCC transceiver nodes consist of a transmitter (TX) and a receiver (RX), together connected to a coupler. Every coupler is composed of two electrodes. For galvanic coupling, these electrodes need to be placed on the skin directly. For capacitive coupling, there is no need for a direct human skin contact [17], unless, then close proximity of the coupler to the body is required. These electrodes can be structured horizontally or vertically where the spacing between them is filled with a dielectric material. These two types of electrode structures are depicted in figure 2.

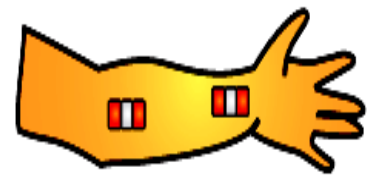

(a) Longitudinal orientation

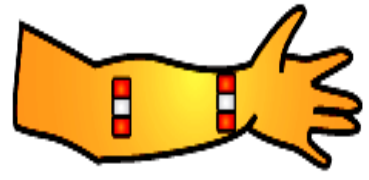

(b) Transversal orientation
Figure 3. The Different Orientations of the Horizontal Coupler Structure

The vertical structure is only used for the capacitive body coupling approach, while the horizontal structure can be applied to both approaches. In figure 3 is noted that in the horizontal structure, the electrodes can be oriented on the body in a longitudinal or transversal direction.

\subsection{The Galvanic Body Coupling}

In this figure 4 the TX an electrical signal is applied differentially between two electrodes that are directly attached to the human body. A current results between these two electrodes. This stimulates a very small electric current to propagate into the conductive body tissues. The RX there are also two electrodes attached to the body and stimulates current results in a differential signal between these two electrodes and are detected by them. This approach makes use of the dielectric characteristics of human tissue, therefore the flow of ions within the human body [18] is the carrier of information and human body acts as a special kind of transmission line.

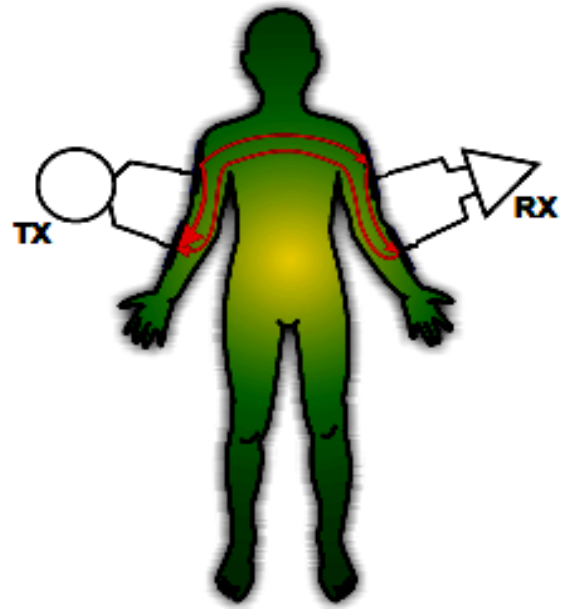

Figure 4. The Galvanic Body Coupling for Data Transmission Between TX and RX

\subsection{The Capacitive Body Coupling}

In capacitive coupling, as it is pictorial in figure 5 a differential pair of electrodes is used both for transmitting and receiving. The

TX side, a signal is applied amid the electrodes and since the electrodes have a dissimilar capacitive coupling to the body, an electric field is stimulated to the human body and passes through the body. The RX side, the two electrodes are at different distances from the body, so it is possible to detect a difference signal between them as a function of the varying electric potential of the person. In this approach, the human body acts as a conductor that forms a bridge between the TX and RX that are capacitively coupled to it. The environment is used as a reference to force or detect a variation of the electric potential of the human body [19].

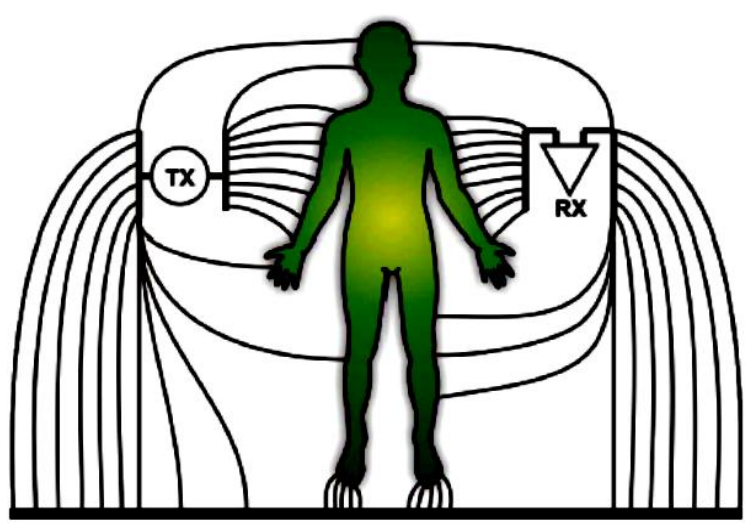

Figure 5. The Capacitive Body Coupling for Data Transmission Between TX and RX

\section{HOW CAN BODY COUPLED COMMUNICATIONS BE USED}

The networks have been made that are basically closing the circuit which is mainly advantageous for safety applications and in between there are security applications and medical body sensor networks. The multimedia and internet packets flow through the body. Until unless there are scenarios where information is shared amid people via physical contact. These are very diverse applications and each of these have very 
diverse requirements. In fact, there are two types of body coupled communications. The first interbody communication [20] and second intrabody communication. The division between interbody and intrabody is the most fundamental differentiator for technical reasons for connecting devices by traversing two people is technically harder to achieve with reasonable performance. In view of the fact that some people might not be compatible with each other and people move which causes the connection to become unstable. The interbody networking also means that two networks could temporary merge which could cause trouble with the software configuration of such networks. Interbody networking deals with the special case that two bodies make physical

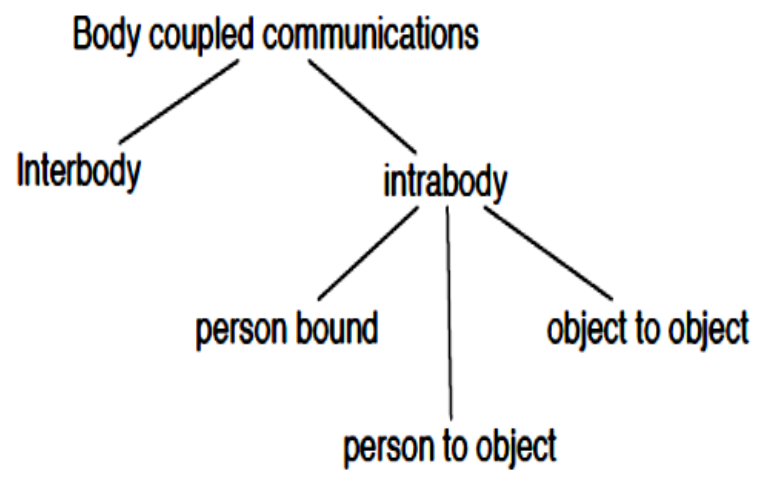

Figure 6. The Communications Hierarchy

contact and information, is sent over the physical connection. For example of such a network would be a network that is created every time somebody gives a handshake and via this handshake

contact information is exchanged. In intrabody communication, there are numerous different usage scenarios. Principally three types of communication can be classified within intrabody communication in figure 6.

- Person Bound Communication

- Person to Object Communication

- Object to Object Communication

As long as the NTT group mention these types of communication in their paper [21], they do not see interbody communication as a different type of communication distinct from intrabody networking. This is strange since they showed that interbody networking was problematic and had different physical properties than intrabody networking. All of these are examples of ubiquitous computing and it should not be too hard to imagine scenarios where all of these types of communication are required.

\section{HOW DOES IT WORK}

The beside body electric-field communication system consists of a transmitter that emits an unstable current (AC) electricfield signal modulated by input data from an electrode and a receiver that uses another electrode to read the weak AC electric field on the body induced by the signal and demodulates it to recover the data from it in figure 7 . The transmitter and receiver are [8] capacitively coupled to the human body through flat electrodes that are equivalent to the antennas in regular wireless systems. The beside the body electric field is modulated and can be read and demodulated through these couplings.

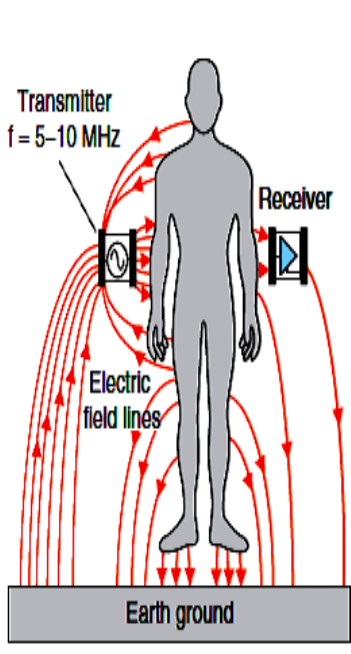

(a) Model of electric-field distribution

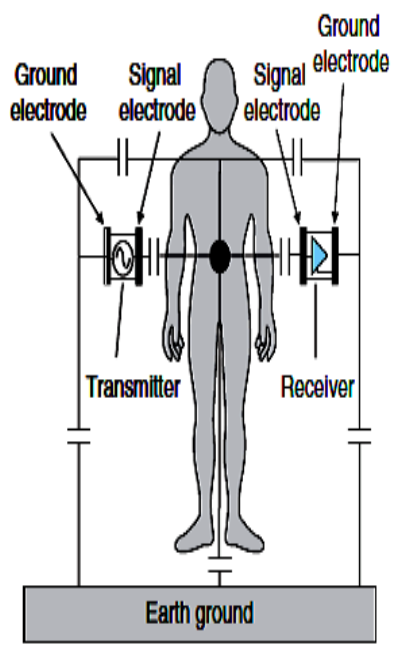

(b) Model of capactive coupling
Figure 7. The Electric-Field and Capacitive-Coupling Models for Near-Body Electric-Field Communications

A specialty of the technology is that, because the signal is passed to and from the body's surface through this capacitive coupling, it can be transmitted between transmitter and receiver via the body's surface, even if one of them is in the user's pocket, and the other is under the carpet on the floor, for example. This technology is applied to a ticket door, it is much more appropriate than appropriate contactless cards because you do not even need to remove the access card from your pocket in figure 8 .

The study this sort of electric-field communications technology, as a means of communication among many wearable computers that users would connect to their bodies [8]. He considers the body as a signal bus between the computers and proposed an electric-field technology using an AC electric field ranging from 0.1 to $1 \mathrm{MHz}$. He constructs a prototype electric-field communications system using 330 $\mathrm{kHz}$ and demonstrated the potential of the concept. The transmission signal is strong, a stronger electric field is emitted around the [22] body, deficiency differentiation from other short-range wireless technologies. In particular the technology is the behavior of the AC electric field, which can be summarized in terms of three components produced around the body when an AC signal is applied to a parallel plate electrode attached to the body. These components, which are all belonging to distance $r$ from the body, are the quasi-static electric field (proportional to $1 / \mathrm{r}^{3}$ ), the induced electric field (proportional to $1 / \mathrm{r}^{2}$ ), and the emitted electric field (proportional to $1 / \mathrm{r}$ ) [22]. The beside body electric-field communications technology uses the quasi-static electric field, which attenuates steeply with $\mathrm{r}$, and we can say that it suppresses both the induced and radiated field components and suppress these emissions, we use a low frequency to drive the transmitter electrode, higher receiver sensitivity, and the minimum required power for the transmitter. 


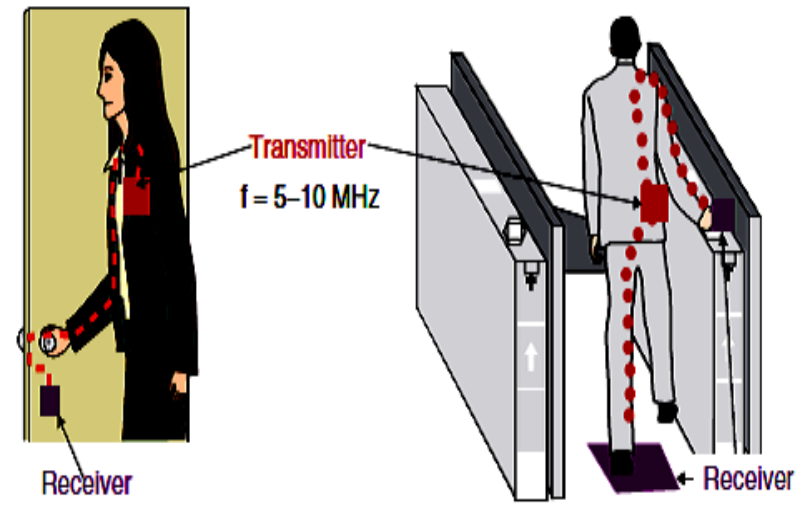

Figure 8. The Applications of Near-Body Electric-Field Communication

Ourselves selected the frequency band from 5 to $10 \mathrm{MHz}$ and developed ways of modulating the near-body electric field efficiently and reducing ambient noise such as electrical humanly in the receiver circuits. This enabled us to develop a near body [22] quasi-static electric-field communications technology adequate of endow the quality required for communications services while using a minimum level of power in the transmitter. The actual electric field offered by our prototype transmitter is an order of magnitude smaller than regulation levels for short-range wireless schemes.

\section{THE LSIS FOR ELECTRIC-FIELD COMMUNICTION}

The uses an alternating current (AC) electric field and are conducting research and development of human body nearfield communication technology. For this technology to come into wide personal use, it must be substantial and inexpensive [5], so the electronic components should be fabricated as LSI (large-scale integration)chips. The main specifications are given in table 1. An LSI to be used in human-body near-field communication must be

\section{Provide two-way communication}

2. Induce an electric-field signal in the human body with benign efficiency

3. To detect very feeble electric-field signals with high sensitivity

4. Mollify environmental noise

5. Have low power consumption

6. Has the same data processing ability and memory capacity as existing IC (integrated circuit) cards

Table 1. The Main Specifications

\begin{tabular}{|l|l|l|}
\hline Analog LSI & Size & $2.5 \mathrm{~mm} \times 2.5 \mathrm{~mm}$ \\
\cline { 2 - 3 } & Carrier frequency & $6.75 \mathrm{or} 9 \mathrm{MHz}$ \\
\cline { 2 - 3 } & Modulation & BPSK \\
\cline { 2 - 3 } & Bit rate & $526 \mathrm{kbit} / \mathrm{s}$ \\
\hline \multirow{3}{*}{ Digital LSI } & Size & $5 \mathrm{~mm} \times 5 \mathrm{~mm}$ \\
\cline { 2 - 3 } & CPU & 16 -bit, $10 \mathrm{MHz}$ \\
\cline { 2 - 3 } & Flash memory & $256 \mathrm{~KB}$ \\
\cline { 2 - 3 } & RAM & $24 \mathrm{~KB}$ \\
\hline
\end{tabular}

\subsection{Analog LSI}

In analog LSI the receiver is high strung to the effects of noise from the environment when the human body is used as a transmission path, we select binary phase shift keying (BPSK) as the synchronization detection method because it endow high noise tolerance [23]. The carrier frequency can be selected as either 6.75 or $9 \mathrm{MHz}$ and the bit rate is about 500 $\mathrm{kbit} / \mathrm{s}$. The chip is $2.5 \mathrm{~mm} \times 2.5 \mathrm{~mm}$, and compact mounting is achieved by using wafer-level packaging. The chip configuration is shown in figure 9. The receiver can switch the data signal demodulation sensitivity and carrier signal detection sensitivity levels, pursuance to the electric field signal strength [24].

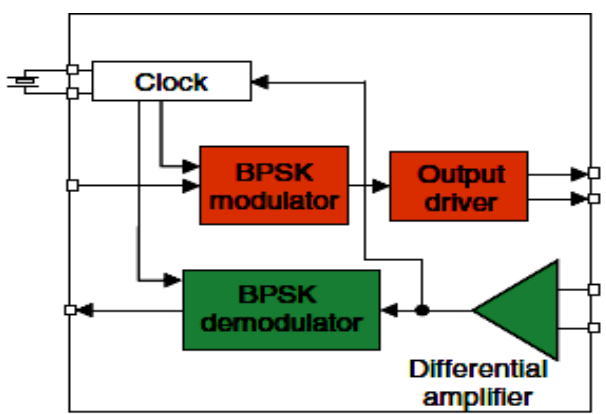

Figure 9. The Analog LSI Configuration

\subsection{Digital LSI}

In digital LSI is shown in figure 10 . We designed the digital chip to provide the same functions as an ordinary IC card. It has a 16-bit central processing unit (CPU) that operates at 10 $\mathrm{MHz}$, a 256-KB Flash memory for storing application software and a 24-KB random access memory (RAM), and a connector for an external 32-KB electrically erasable programmable read-only memory (E2PROM) for IC card data.

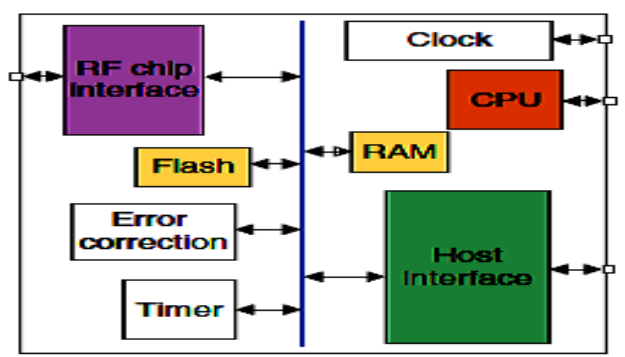

Figure 10. The Digital LSI Configuration

It measures $5 \mathrm{~mm} \times 5 \mathrm{~mm}$ and, like the analog LSI, uses wafer-level packaging for compact

mounting. Since portable devices are battery driven [24], LSI chips designed to be mounted on portable devices must have low power consumption.

\section{THE REDTACTON TRANSCEIVER}

The block diagram of a RedTacton Transceiver shown in figure 11. The transmitter to be composed of a transmitter circuit that induces electric fields toward the body and a data sense circuit, which differ transmitting [25] and receiving modes by detecting both transmission and reception data and outputs control signals commensurate to the two modes to enable two way communication in figure 12 . The receive-first 
half-duplex communication scheme that sends only after checking to constitute that there is no data to receive in order to avoid packet collisions between terminals in compliance with the IEEE 802.3 protocol.

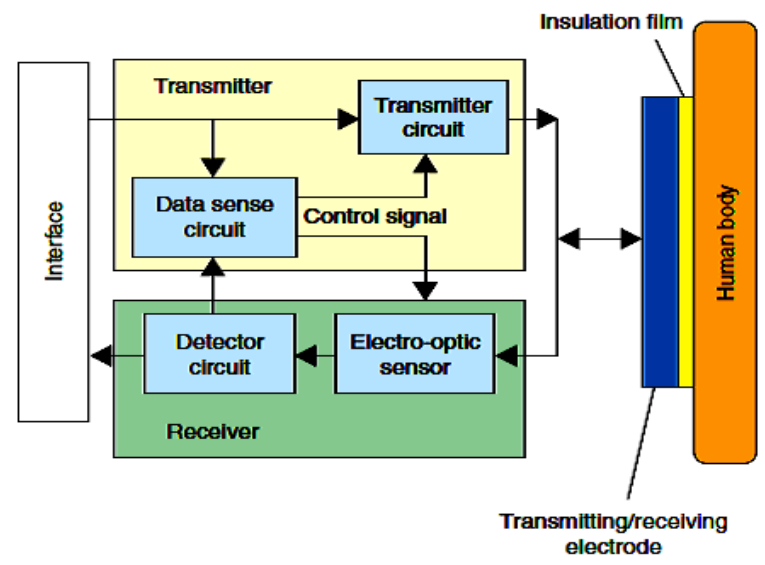

Figure 11. The RedTacton Transceiver

The receiver consists of an electro-optic sensor and a detector circuit that amplifies the minute incoming signal from the an electrooptic sensor and converts it to an electrical signal. Ourselves conducted a series of trials in which data was sent through human bodies using RedTacton transceivers. The experimental setup for intrabody communication assuming communication between two electronic devices (PDAs) is shown in figure 13. We produce two sets of RedTacton transceivers, each connected to a PDA. The subject held one transmitting, receiving electrode in each hand [5]. We quantitatively measured the bit error rates of signals sent through the body. In addition, communication between two hands, we also demonstrated authentic communication between a foot and finger and between other locations on the person's body.

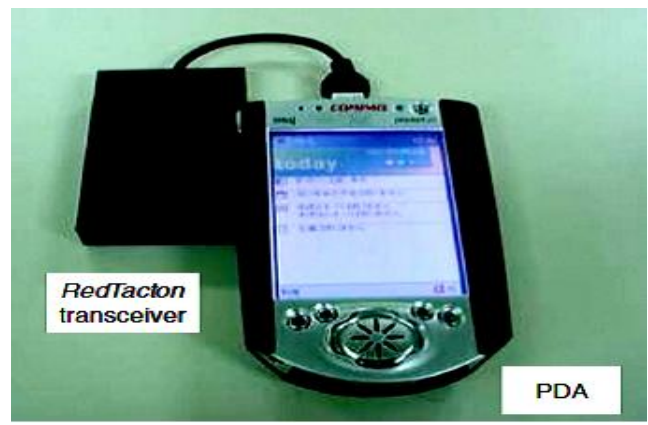

Figure 12. The RedTacton Transceiver and PDA

We also calibrate that good communication was achieved not [25] only when the electrodes were in direct contact with the person's skin, but also when the signals passed through clothing and shoes.

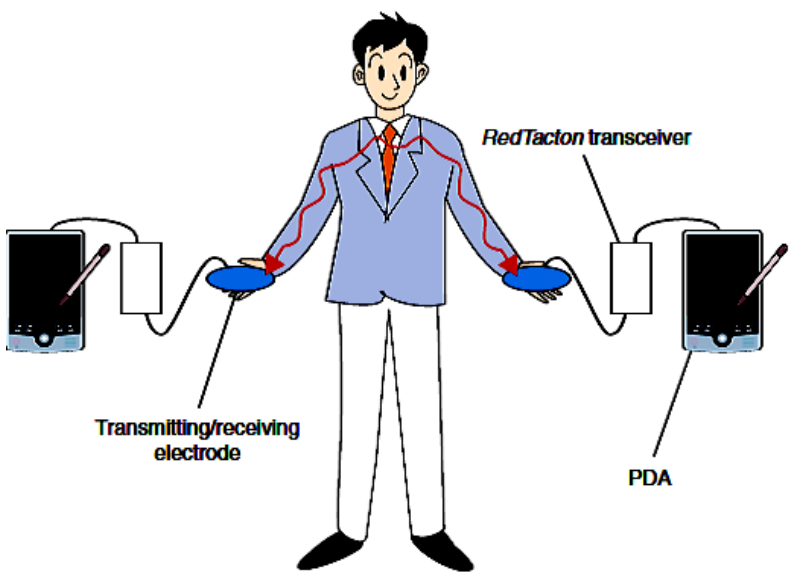

Figure 13. The Experimental Setup for Intrabody Communication

\section{THE COMMUNCTION DEVICES REDTACTON MODULE}

There are three kinds of communication devices (card, mobile, embedded terminal) that can have a RedTacton module in table 2 .

\subsection{Card Terminal}

The thin device in card form. The power is supplied by a compact battery, the need for low power consumption in particular important. The card terminal can provide the same services as existing IC (integrated circuit) cards, it must have a [26] file management function for managing data for authentication or certification and card terminal works without a supervising device, it is a slave in the masterslave communication model.

\subsection{Mobile Terminal}

The component built into a mobile device, such as a cell phone. It in state power from the built-in battery of that device. It is used for the same services as IC cards or for services using communication between mobile terminals, a mobile terminal must

Table 2. The Communication Devices RedTacton Module

\begin{tabular}{|c|c|c|c|c|c|}
\hline Type & Power supply & Power saving & Fllemanaagement & Supervising device & Communiatalon olde \\
\hline $\begin{array}{ll}\text { Card } & \\
\text { temminal } & -\end{array}$ & Battery & Required & Requiled & Not required & Slave \\
\hline $\begin{array}{ll}\text { Molle } & d \\
\text { temmanal } & i\end{array}$ & Batten & Required & Required & Required & Naster, save, peer \\
\hline $\begin{array}{l}\text { Enbedded } \\
\text { temminal }\end{array}$ & Mains AC supply & Notrequired & Notrequired & Required & Masser, peer \\
\hline
\end{tabular}

have a file management function and a function for connecting to a supervising device. A mobile terminal works under a supervisor and has a file system. It has three roles either a master or slave in masterslave communication or a peer in a peer-to-peer communication in other words (in which control between the terminals is on an equal basis). As a slave, it communicates with other embedded terminals or 
mobile terminals (acting as masters) and as a master it communicates with other mobile terminals (acting as slaves).

\subsection{Embedded Terminal}

The device built into a door or gate. It is powered by mains AC, low power consumption is not an important issue. Although an embedded terminal does not need to have a file management function in order for the gate management server to keep user data, it needs to have a function for connecting to a supervisor [26]. The embedded terminal works with a supervisor, it is the master in master-slave communication or a peer in a peer-to-peer communication.

\section{THE STRUCTURE OF REDTACTON FIRMWARE}

The structure of the firmware that meets the requirements is shown in figure 14. There in the multichip module is another name for a RedTacton module from the hardware viewpoint. Firmware is located in the digital LSI (large-scale integrated circuit) part of the RedTacton module and has an electric-field communication interface and a wired-communication interface. The electric-field communication interface is the gateway [26] to another RedTacton module through the analog LSI part of the RedTacton module. The wiredcommunication interface is the gateway to a supervising device.

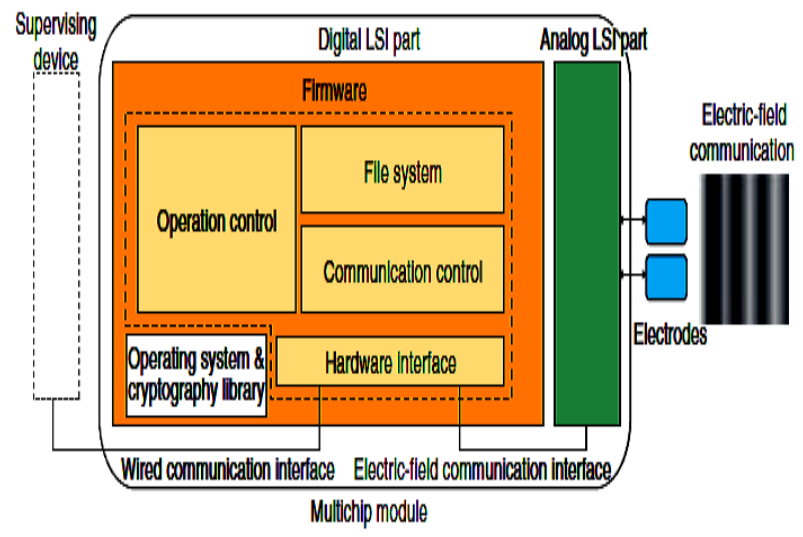

Figure 14. The Structure Of RedTacton Firmware

\section{THE FUNCTIONAL FEATURES OF REDTACTON}

Red Tacton has a wide range of unprecedented new functional features and enormous potential as a Human Area Networking technology [3]. The RedTacton is a big achievement given by NTT to people. The RedTacton has three main functional features as shown in figure 15 .

\subsection{Touch}

This is the prime feature of RedTacton. A communications path can be created with a simple touch, automatically initiating the flow of data between a body-centric electronic device and a computer that is embedded in the environment. For example, a

simple handshake between people having RedTacton device, sitting, stepping on something and so on.

\subsection{Broadband \& Interactive}

The second feature is about little interference or no interference. The RedTacton reliance communication between two points on the body, regardless that the communication must be confined to the surface of the body, but can travel through the user's clothes, shoes and so on to the RedTacton device. The dissimilar wireless technologies, the transmission speed do not vary even in the presence of large crowds of people all communicating at the same time in meeting rooms, auditoriums or stores. The duplex, interactive communication is possible at a maximum speed of $10 \mathrm{Mbps}$. Therefore the transmission path is on the surface of the body, transmission speed does not deteriorate in congested areas where many people are communicating at the same time. Hence the body surface is the transmission path, increasing the number of connected users directly increases the available number of individual communication channels.

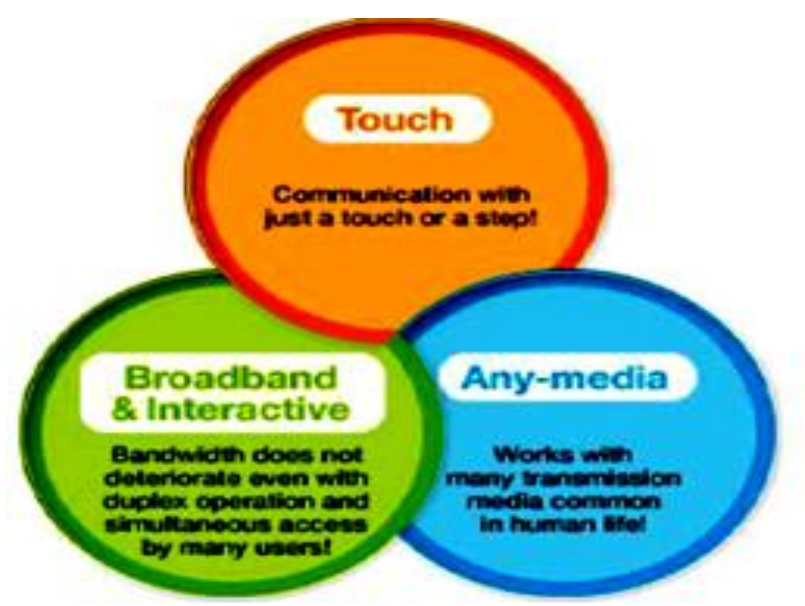

Figure 15. The Functional Features Of RedTacton

\subsection{Any Media}

The third feature is any media meaning that the transmission medium is not immobile. A wide variety of materials can be used as the transmission medium, meanwhile the material is conductive and dielectric, which includes water and other liquids, various metals, certain plastics, glass. The ordinary structures such as tables and walls that are familiar and readily available, one could easily construct a consolidated communication environment at very low cost using RedTacton

\section{THE POTENTIAL APPLICATIONS OF REDTACTON}

There are many potential applications of RedTacton in different fields. This technology will widely use in daily working schedule and endow facility to people. There are seven major potential applications that are done using RedTacton [27]

\subsection{One to One Services}

Enable one-to-one services tailored to the user's situation and tastes. Attribute information recorded in the RedTacton device is sent to the touched objects. The appropriate service is provided based on the attribute information received by the RedTacton receiver. 


\subsubsection{Elimination of Human Errors}

RedTacton devices embedded medicine bottles transmit information on the medicines' attributes. If the user touches the erroneous medicine, an alarm will trigger on the terminal he is carrying. The alarm sounds only if the user actually touches the medicine bottle, reducing false alarms common with passive wireless ID tags, which can trigger simply by proximity.

\subsubsection{Marketing Applications (Context \\ Awareness)}

When a consumer stands in front of an advertising panel, advertising and information matching his or her attributes are automatically displayed. Through touching or standing in front of items they are interested in, consumers can get more in-depth information.

\subsection{Intuitive Operations}

There's no operation any more. Just intuitive human interaction. The RedTacton transceivers embedded in two terminals can communicate not only data but also the control or configuration instructions needed to operate devices [27]. The body itself is used as a transmission medium. Example for Print out where you want just by touching the desired printer with one hand and a PC or digital camera with the other hand to make the link. The sophisticated configurations are reduced by downloading device drivers at first touch.

\subsubsection{Instant Private Data Exchange}

By shaking hands, personal profile data can be exchanged between mobile terminals for the users. (Electronic exchange of business cards). The communication can remain private using authentication and encryption technologies.

\subsection{Personalization}

The digital lifestyle can be instantly personalized with just a touch. A pre-recorded configuration script can be embedded in a mobile terminal with a built-in RedTacton transceiver. When another device with RedTacton capabilities is touched, personalization data and configuration scripts can be downloaded automatically.

\subsubsection{Personalization of Mobile Phones}

Your own phone number is allocated and billing commences automatic importing of personal address book and call history.

\subsubsection{Personalization of Automobiles}

The seat position and steering wheel height adjust to match the driver just by sitting in the car. The driver's home is set as the destination in the car navigation system. The stereo plays the driver's favorite song.

\subsection{New Behavior Patterns}

Various conductors and dielectrics can be used as RedTacton communication media, and this has the potential to create new behavior patterns. The RedTacton can carry music or video between headsets, mobile devices, mobile phones, etc. Users can listen to music from a RedTacton player simply by putting on a headset or holding a viewer.

\subsection{Security Applications}

Automatic user authentication and log-in with just a touch. ID and privileges are recorded on a mobile RedTacton device. At the RedTacton receivers are installed at security checkpoints
[4]. The system can provide authentication and record that touched the device, and when carrying a mobile RedTacton capable device in one's pocket, ID is calibrated and the door unlocked when the user holds the doorknob normally. Secure lock administration is possible by combining personal verification tools such as fingerprint ID or other biometric in the mobile terminal.

\subsubsection{Confidential Domain Management}

A RedTacton receiver in a cabinet where important documents are stored enables lock administration and keeps a record of who accessed documents at what time.

\subsection{Communication in New Domains}

RedTacton allows communication in outer space and in water where the speech constraints are very high and thus enables a highly efficient means of expression of speech which is beyond the purview of human. RedTacton is also used for the treatment. In the human body, it is used to detect ailments such as abnormal growths, tumors and excrescences affected tissues and thus helps in curing different diseases.

\subsection{Military Applications}

In the military applications During the war, a soldier having a Red Tacton authenticated gun is supposed to fire with that respective gun. Nobody soldier can use that gun for firing if that authenticated soldier is dead or not able to participate in the war. It gives advantages that enemy soldier cannot attack the other party with their own ammunitions. This can detract the black market of weapons and misuse of stolen weapons, especially the misuse by the children.

\section{ADVANTAGE \& DISADVANTAGE OF REDTACTON}

\subsection{Advantage of RedTacton}

The RedTacton does not require the electrode be in direct contact with the skin. The high-speed communication is possible between two arbitrary points on the body. In Bodybased networking is more secure than broadcast systems, such as Bluetooth which have a high range of about $10 \mathrm{~m} \mathrm{[27].} \mathrm{In}$ the Network congestion due to fall in transmission speed in multiuser environments is avoided. The preferable than Infrared technology and superior than Wi-Fi. The data transfer during transfer is less and use a minimum amount of power. The electric amperage method, a clear signal line and ground line are required. With the electric voltage method, capacity coupling can substitute.

\subsubsection{Advantages Over Bluetooth and RFID}

This way the technology is clearly advantageous over Bluetooth and RFID in the following factors. The traffic and Network congestion does not occur here and it decreases with an increase in the number of users joining the network. It highly supports interactive processing. It greatly reduces the power consumption and cost of transmission media under usage. The data transfer rates are very impressive and it ranges to maximum of 10

Megabits per second. It turns our body into a swipe card which can be applied to security purposes. It can be applied for security purposes in a variety of domains. RedTacton does not require the electrode be in direct contact with the skin. 


\subsection{Disadvantage of RedTacton}

It has no compelling applications that aren't already available. It is too costly. It can be useful within centimeters and effects on the human body is still under research. It takes more time to get popular among the people in the market as it is a new kind of network.

\section{THE COMPARISON WITH OTHER NETWORKS}

The chart below shows the positioning of RedTacton with respect to existing communication technologies. The focus on ubiquitous service has brought about the shortening of distances in communication The chart below shows the positioning of RedTacton with respect to existing communication technologies. The fact that communication is only with the touched object, we compared it with other popular short-range wireless and contactless-card technologies in terms of transmission speed and propagation distance through space figure 16. RedTacton limits the propagation through space to a range similar to contactless cards and targets transmission speeds from $100 \mathrm{kbit} / \mathrm{s}$ to 10 Mbit/s. In contrast to technologies like wireless local area networks, Bluetooth, [28] and ZigBee, which can propagate signals for over $10 \mathrm{~m}$ through space, communication is limited to devices within arm's reach. In addition to the above characteristics, in RedTacton [29], the start and end of communication are linked to the user's actions, which gives an intuitive sense of connection to the network. This makes the technology a universal data transmission interface, with the potential for expanding the boundaries of information and communications technology (ICT) and creating whole new application areas.

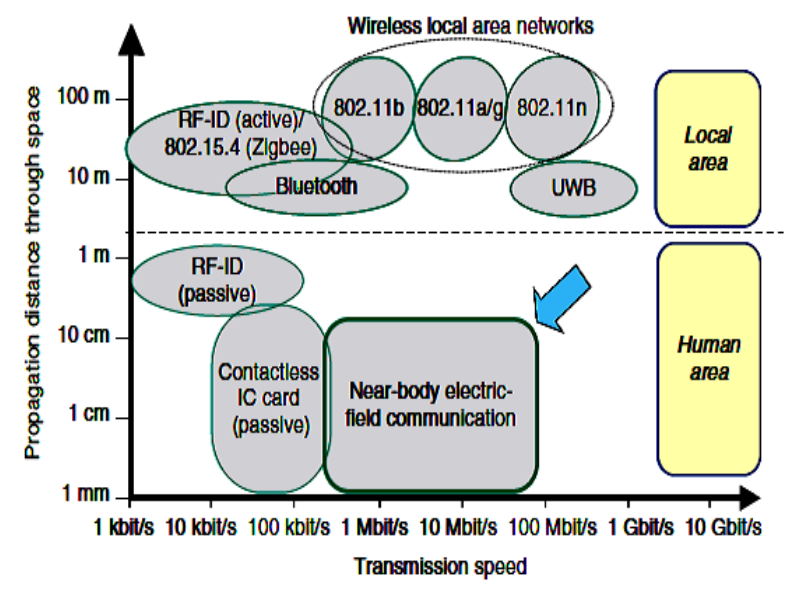

Figure 16. The Comparison With Other Networks

\section{THE FUTURE DEVELOPMENTS}

The RedTacton has a wide range of unique new functional features and enormous potential as a Human Area Networking technology. RedTacton is a big achievement given by NTT to people. NTT is committed to quickly identifying and opening up those application areas with the most commercial promise for Red Tacton is a business development process to be coordinated under NTT's Comprehensive Product Function program shown in figure 17. RedTacton looks remarkably like a big pot of kryptonite is said to allow [29] over 200kbps of data through th10 human hands or feet. Telecom giant Nippon Telegraph and Telephone Corp (NTT) is planning a commercial launch of a system to enter rooms that frees users from the trouble of rummaging in their pockets or handbags for ID cards or keys. The RedTacton has a wide range of unique new functional features and enormous potential as a Human Area Networking technology. NTT is committed to quickly identifying and opening up those application areas with the most commercial obligation for RedTacton, a business development process to be coordinated under NTT's.

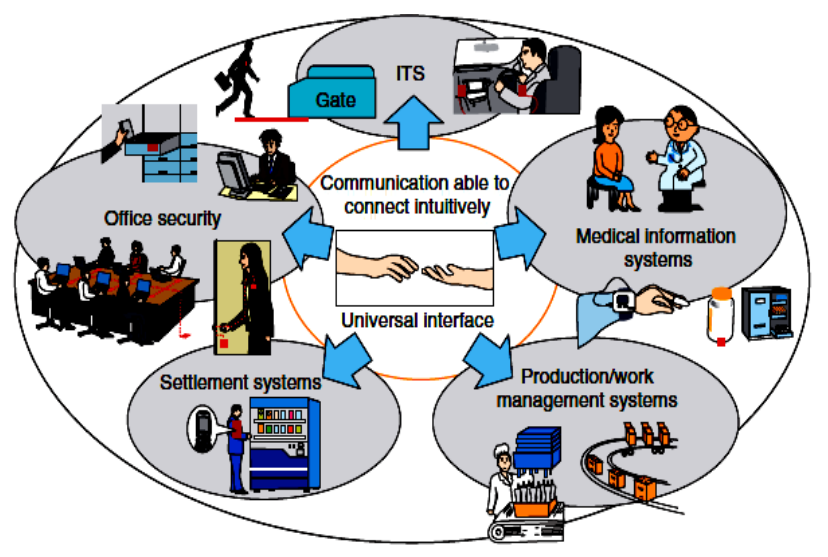

Figure 17. The Expected Applications Areas

\section{CONCLUSION}

The principle deficiency of infrared communications (IrDA) is the tight directionality of beams between terminals needed for the system to be influential. The optimum human area network solution to all these constraints of traditional technologies is intrabody communication, in which the human body serves as the transmission medium. If we could use the human body itself as a transmission medium, then this would be a surpassing way of implementing human area networks because it would extricate at a stroke all the problem reduction, low security, congestion, and high network setup costs. The consideration of including throughput intrabody communication, which uses the minute electric field propagated by the human body to transmit information. The RedTacton is an exciting new technology for human area networking. We have developed a transceiver that uses the human body as a data transmission medium based on an electric-field sensor that uses an electro-optic crystal and laser light. RedTacton technology is expected to kingship Bluetooth technology in the future. This technology brings a state-ofthe-art dimension of communication which effectively links the user to anyone he wants to communicate. It provides towering speed communication, it can provide seamless service wherever, whenever and whoever uses it. I conclude that, when I differentiate RedTacton with other technology present today it can give a preferable performance over others. I can say that to connect the network within short distances RedTacton is best. With this technology, there is no problem of hackers as our body is itself a medium. This RedTacton can be used as a substitute to present day networking technologies which are limited by traffic congestion and data loss. The Future proposal in this field of networking is that I can embed Artificial Intelligence and Neural Networking concepts which take RedTacton a step further. Therefore, the Human Area Networking sets a wide horizon for upcoming data transfer technologies. As discussed above, no other technology can substitute substitute and finally, I conclude future belongs to the RedTacton 


\section{REFERENCES}

[1] Wikipedia.(February 2009). Red Tacton [Online]. Available: http://en.wikipedia.org/wiki/RedTacton

[2] http://www.redtacton.com/en/index.html

[3] Kotadia, B.; Vibhor, A.; "REDTACTON", Electronics \& Communication Department, MIT IEEE Report http://www.scribd.com/doc/5007416

[4] Redtacton-IEEE.ReportTechnicalpapers.50webs. $\mathrm{http} / / /$ technicalpapers.50webs.com/pdf/redtaction.pdf

[5] M. Shinagawa, M. Fukumoto, K. Ochiai, and H. Kyuragi, "A nearfield- sensing transceiver for intra-body communication based on the electro-optic effect," IEEE Trans. IM, Vol. 53, No. 6, pp. 1533-1538, 2004.

[6] Kohei Nagata, Yuichi Kado, "Transmission Characteristics between In-body and On-body Transceivers Using MHz-band Near-field Coupling Technology" ,IEEE, 7th International Symposium on Medical Information and Communication Technology (ISMICT) 2013.

[7] Simon Attard, Saviour Zammit," Effect of human movement on the Body Coupled Communications channel in Body Area Networks",IEEE, 1-4 July 2013.

[8] T. G. Zimmerman, "Personal Area Networks: Near-field intrabody communication,"IBM Systems Journal, vol.35, no.3.4, pp.609,617, 1996.

[9] N. Matsushita, S. Tajima, Y. Ayatsuka, and J. Rekimoto. Wearable key: Device for personalizing nearby environment. In ISCWC00, 2000.

[10] H.J. Yoo, S.J. Song, N.Cho, and H.J. Kim. Low energy on-body communication for bsn. In Body sensors networks 2007.

[11] J. Hwang, J. Sung, C. Hyoung, J. Kim, D. Park, and S. Kang, "Analysis of signal interference in human body communication using human body as transmission medium," in IEEE Antennas and Propagation Society International Symposium 2006., July 2006, pp.495-498.

[12] A. Sasaki, M. Shinagawa, and K. Ochiai. Principles and demonstration of intrabody communication with a sensitive nelectrooptic sensor. IEEE Transactions on instrumentation and measurement, 58(2), february 2009.

[13] L.Cho, L.Chan, J.Bae, and H.Yoo. A $60 \mathrm{~kb} / \mathrm{s}-10$ $\mathrm{mb} /$ sadaptive frequency hopping transciever for interferance-resilient body channel communication. IEEE Journal of Solid state circuits, 44(3), March 2009.

[14] Baldus, H.; Corroy, S.; Fazzi, A.; Klabunde, K.; Schenk, T., "Human-centric connectivity enabled by bodycoupled communications,"Communications Magazine, IEEE, vol.47, no.6, pp.172,178, June 2009.

[15] Patel, M.; Jianfeng Wang, "Applications, challenges, and prospective in emerging body area networking technologies," Wireless Communications, IEEE , vol.17, no.1, pp.80,88, February 2010.

[16] Wegmueller, M.; Lehnert, A.; Froehlcih, J.; Reutemann, R.; Oberle, M.; Felber, N.; Kuster, N.; Hess, O.; Fichtner, Wolfgang, "Measurement System for the
Characterization of the Human Body as a Communication Channel at Low Frequency,"Engineering in Medicine and Biology Society,. IEEE-EMBS 2005. 27th Annual International Conference of the, vol., no., pp.3502,3505,

[17] T. Schenk, N. S. Mazloum, L. Tan, and P. Rutten, "Experimental characterization of the body-coupled communications channel," in ISWCS 2008, October 2008.

[18] Wegmueller, M.S.; Kuhn, A.; Froehlich, J.; Oberle, M.; Felber, N.; Kuster, N.; Fichtner, Wolfgang, "An Attempt to Model the Human Body as a Communication Channel,"Biomedical Engineering, IEEE Transactions on, vol.54, no.10, pp.1851,1857, Oct. 2007.

[19] M. Fukumoto, M. Shinagawa, K. Ochiai, and H. Kyuragi, "A near-field-sensing transceiver for intrabody communication based on the electrooptic effect," in IEEE Transactions on Instrumentation and Measurement, vol. 53, December 2004, pp. 1533-1538.

[20] T.C.W. Schenk, N.S. Mazloum, L. Tan, and P. Rutten. Experimental characterization of the body-coupled communications channel. In International Symposium onWireless Communication Systems (ISWCS 2008), 2008.

[21] M. Shinagawa, K.Ochiai, H.Sakamoto, and T.Asahi.Human area networking technology: Redtacton. NTTReview, 3(5), May 2005.

[22] H. Goldstein, "NTT's shaky approach to data transfer targets a solved problem," IEEE Spectrum January 2006, pp. 24-25, 2006.

[23] K. Doi, M. Koyama, Y. Suzuki, and T. Nishimura, "Development of the communication module used human body as the transmission line," [sic] Proc. of Human Interface Symposium 2001, pp. 389-392, 2001 (in Japanese).

[24] Ryusuke Kawano, Fumiaki Morisawa, Mitsuru Shinagawa, and Yuichi Kado," LSIs for an Advanced Compact Electric-field Communication Module", NTT Microsystem Integration Laboratories Atsugi-shi, 2430198 Japan, Vol. 8, No. 3, Mar. 2010.

[25] Mitsuru Shinagawa, Katsuyuki Ochiai, Hideki Sakamoto, and Toshiaki Asahi," Human Area Networking Technology: RedTacton", NTT Microsystem Integration Laboratories, Vol. 3, No. 5, May 2005

[26] Hideki Goromaru, Minoru Ikeda, and Yasuhiro Hosoda," Firmware Technology for Human-body Near-field Communication”, NTT Technical Review, Vol. 8, No. 3, 2010

[27] Saheed A. Adewuyi, Idowu O. Aiyedun, Oluwafemi T. Balogun," RedTacton: Enhancing Ubiquitous Computing Services", Proceedings of the World Congress on Engineering 2013 Vol II, WCE 2013, July 3 - 5, 2013, London, U.K.

[28] Yusuf Perwej, Dr. K. Haq, U. Jaleel , S. Saxena , "Some drastic improvements found in the analysis of routing protocol for the Bluetooth technology using scatternet" Special Issue on The International Conference 
on Computing, Communications and Information Technology Applications (CCITA-2010) , Ubiquitous Computing and Communication Journal (UBICC) Seoul, South Korea, Volume CCITA-2010, Number 5 , pages 86-95, ISSN Online 1992-8424, ISSN Print 19944608.

[29] Yuichi Kado, Mitsuru Shinagawa," RedTacton Nearbody Electric-field Communications Technology and Its Applications", NTT Technical Review, Vol. 8, No. 3, 2010.

\section{AUTHOR PROFILE}

Dr. Yusuf Perwej Assistant Professor in the Department of Computer Information System Al Baha University, Al Baha, Kingdom of Saudi Arabia (KSA). He has authored a number of different journal and paper. His research interests include Soft Computing, Artificial Neural Network, Machine Learning, Pattern Matching, Pattern Recognition, Artificial Intelligence, Image Processing, Fuzzy Logic, Genetic Algorithm, Robotics, Bluetooth and Computer Network etc. $\mathrm{He}$ is a member of IEEE. 